\title{
NARRER LES DÉSILLUSIONS INTIMES À L'ÉPOQUE DE LA DÉSILLUSION. TANGUY VIEL ET LES FORMES CONTEMPORAINES DU RÉCIT D'AMITIÉ
}

Le motif des relations humaines est celui qui confère à l'œuvre littéraire de Tanguy Viel sa véritable et profonde unité. Toutes ses œuvres soulèvent les questions des liens qui unissent les gens, de leurs formes et influences psychologiques sur la vie de l'individu. Parmi ces relations, l'amitié occupe une place de choix et se voit interrogée de façon à la fois dramatique et poétique surtout dans ses trois premiers textes qui forment une sorte de trilogie romanesque ${ }^{1}$. Pour investir ce motif tant de fois débattu dans la littérature et les arts, l'écrivain conjugue les variations génériques et une narration non-fiable, modalités propres à la littérature récente. En nous appuyant sur les théories narratologiques contemporaines, nous chercherons à étudier ces deux aspects des romans de Viel pour montrer la manière tout à fait ingénieuse dont ils véhiculent la thématique soulevée. Notre analyse sera également nourrie de quelques conceptions philosophiques de l'amitié — notamment celles d'Aristote, Maurice Blanchot et Jacques Derrida au sujet de l'amitié interpersonnelle, ses formes et fondements, ainsi que celles de Marcel Proust et John Dewey sur l'amitié esthétique, à savoir une relation affectueuse qui se tisse entre l'homme et un objet d'art dans le processus de réception - conceptions essentielles dans une réflexion particulièrement abondante sur la problématique de l'amitié et qui semblent les plus opératoires pour saisir le sens des romans de Viel.

${ }^{1}$ T. Viel, Le Black Note, Minuit, Paris 1998 (désigné dans le texte par le sigle BN); idem, Cinéma, Minuit, Paris 1999 (désigné par C) ; idem, L'Absolue perfection du crime, Minuit, Paris 2001 (désigné par $A P C$ ). 
Ce qui fait l'originalité de cette trilogie amicale, c'est surtout sa structure romanesque où se laisse voir le processus de « démolitions des formes littéraires $»^{2}$ propre aux textes contemporains. Représentant de la jeune génération de Minuit, Tanguy Viel investit l'écriture subversive des genres qui fait la marque stylistique des écrivains édités par la célèbre maison d'édition après les années 1980. Jean Echenoz surtout, mais aussi Jean-Philippe Toussaint et Éric Chevillard se sont fait connaître justement par des romans génériquement « indécidables » ${ }^{3}$, détournant les schémas d'intrigue et la dynamique fictionnelle traditionnels. Viel procède de manière analogue, jouant avec cette grammaire idéale du texte qu'est le genre littéraire. Inscrits dans le contexte de la culture populaire, ses romans actualisent des structures littéraires particulièrement captivantes pour le lecteur pour s'en distancier aussitôt par toute une série de procédés. Cette forme claudicante, pâle reflet du modèle canonique, se fait porteuse d'une histoire d'amitié dont le sens se crée surtout à travers des dérogations génériques intentionnelles.

« Classic thriller genre ${ }^{4}$, Le Black Note est un polar qui tient le lecteur en haleine jusqu'au dénouement malgré d'évidentes prolepses inadmissibles dans le roman de détection traditionnel. Car elles sont très adroitement affectées d'incertitude et maintenues dans le régime de l'hypothétique de sorte qu'elles ne peuvent pas être interprétées comme des faits indubitables. Conformément aux règles du genre, le roman débute par un crime dont le récit promet d'élucider les circonstances et les motifs, l'identité du criminel étant dévoilée dès les premières lignes du texte. C'est bien le narrateur qui, traumatisé par l'incendie dans lequel est mort son meilleur ami, se retrouve à l'hôpital psychiatrique pour une cure médicale, mais aussi en observation en vue de déterminer la vraie nature de l'incident survenu. L'enquête qui se déroule n'a rien à voir avec un déchiffrement d'indices par un détective, mais prend la forme d'une investigation intérieure effectuée par le supposé coupable. L'effort mémoriel qu'elle lui demande est énorme, car le passé s'embrouille dans sa conscience ébranlée par le traumatisme vécu et s'efface progressivement sous l'influence des médicaments sédatifs. Or, si « la mémoire n'est jamais panoptique chez Viel $»^{5}$, c'est non seulement en raison de son essence même mais aussi parce que les souvenirs sont refoulés ou intentionnellement passés sous silence à cause des remords de conscience :

2 J. Mecke, « Démolition de la littérature et reconfiguration post-littéraire », [dans :] W. Asholt, M. Dambre (dir.), Un retour des normes romanesques dans la littérature française contemporaine, Presses Sorbonne Nouvelle, Paris 2010, p. 39.

${ }^{3}$ B. Blanckeman, Les récits indécidables : Jean Echenoz, Hervé Guibert, Pascal Quignard, Presses Universitaires du Septentrion, Paris 2000, p. 13.

${ }^{4}$ A. Blatt, «Tanguy Viel's Manic Fictions », Contemporary French \& Francophone Studies XIV, 4, 2010, p. 373.

${ }^{5} \mathrm{Ch}$. Mercandier, «Les lois de l'abstraction : le blanchiment du noir chez Julia Deck et Tanguy Viel », Revue critique de fixxion française contemporaine 10, 2015, p. 119. 
Je ne tiens plus avec ma conscience. C'est comme si je délirais encore plus, mais cette fois il y a la vérité qui sue par les pores de mes joues quand je parle et je peine, Georges, je peine à tout contenir. C'est fini maintenant, et c'est seulement dans ma tête à moi, n'est-ce pas, dans notre tête à tous les deux. Mais un jour, je raconterai tout. (BN, p. 89)

L'anamnèse, qui sert ici de ressort textuel, aboutit à un récit forcément incomplet et contradictoire qui fait ressortir plus « des nœuds dans l'affaire, des points noirs » $(B N$, p. 61) qu'il ne les élucide.

Ces bribes laissent pourtant déduire les raisons du crime qui semble être une variante d'acte passionnel causé par un désenchantement profond de type relationnel. Ils étaient quatre jeunes hommes réunis par un amour pour le jazz, une vraie famille musicale créée sous l'impulsion de leurs affinités d'âme. Or, les sept années passées à composer et répéter des mélodies dans une maison appelée symboliquement Black Note - ainsi s'exprime, selon Aristote, l'amitié parfaite qui est « le choix réfléchi de vivre ensemble [...] en vue des belles actions ${ }^{6}$ n'ont pas laissé se former de véritables liens entre eux et encore moins réaliser ces grands objectifs que le philosophe considère possibles par la force de la relation. Car la véritable amitié est plus qu'une existence commune, côte à côte et au jour le jour, et elle ne peut s'épanouir qu'à travers une profonde acceptation de l'autre dans sa différence. Maurice Blanchot n'est pas le seul à insister sur ce point quand il considère l'amitié comme une « communauté d'égaux [...], telle qu'elle ne les subordonne pas les uns aux autres $»^{7}$. Sous l'influence de Paul qui ne se satisfait pas de jouer de la musique mais ambitionne de recréer le quartette de jazz de Coltrane, l'amitié dépérit pour évoluer vers la domination. Au lieu de permettre cet épanouissement personnel que les philosophes considèrent comme l'avantage majeur des liens affectifs unissant les gens, elle entraine une destruction identitaire. Censés incarner parfaitement leurs idoles, les habitants de Black Note vont jusqu'à s'identifier à eux en empruntant leurs noms et apparence. Dominés par le simulacre créé, ils ne vivent plus désormais qu'une fausse vie, confondant l'illusion et la réalité :

[Paul] délirait à nouveau, et se persuadait que c'était le vrai saxophone, le vrai John Coltrane qui le lui avait offert [...]. Il a toujours cru qu'on y croyait [...]. Finalement c'est devenu vrai, parce qu'il faisait comme si c'était pour de vrai, et on faisait comme si c'était pour de vrai, et du coup c'est devenu vrai, à cause du double mensonge. (BN, p. 23)

L'incapacité du narrateur à satisfaire le fantasme de Paul entraîne l'exclusion de celui-ci de la communauté amicale : trompettiste et non pianiste, il est cette faille qui empêche la concrétisation idéale du grand projet. Ainsi, pour citer Alice Richir, «l'absence de masque derrière lequel se projeter [...] cause son aliénation ${ }^{8}$. Si ce rejet le fait profondément souffrir et l'isole, il ne constitue cependant

\footnotetext{
${ }^{6}$ Aristote, Les Politiques, Garnier Flammarion, Paris 1993, p. 237.

${ }^{7}$ M. Blanchot, La communauté inavouable, Minuit, Paris 1983, pp. 14-15.

${ }^{8}$ A. Richir, « Faire jazzer la voix narrative », Relief 6 (2), 2002, p. 73.
} 
pas le motif direct du crime qui tient plutôt d'un acte de solidarité amicale que d'une vengeance personnelle. Non impliqué dans le jeu de rôles, le narrateur voit mieux que les autres la dégringolade où ils ont tous sombré à cause de Paul. Car la passion qui les a unis s'évapore, les musiciens ne jouent plus, passant des journées entières à ne rien faire d'autre qu'attendre quelque stimulus de la part de leur leader qui dévie de la réalité, plongé dans des illusions artificielles créées au moyen des drogues et de l'alcool. La conscience du malheur inévitable pousse le narrateur à mettre fin à cette farce tragique pour éviter à ses amis et à lui-même cette lente agonie que Paul expérimente de son vivant.

Avec Cinéma, Viel investit le genre assez peu répandu qu'est la novellisation, à savoir la transformation d'une œuvre visuelle en un livre. Fidèlement calqué sur l'original cinématographique que le lecteur averti reconnaît sans peine dès le début de l'action, le roman se présente comme une version romancée de Sleuth (1972), dernière réalisation de Joseph Mankiewicz. Dans sa forme classique, la novellisation ne recourt pas aux techniques narratives empruntées au langage du cinéma, mais elle est une fiction de facture plutôt conventionnelle, censée transposer en récit écrit l'histoire narrée à l'origine au moyen d'images. Chez Viel pourtant, le transfert du film en littérature ne s'effectue pas conformément aux règles du genre, et ceci principalement à cause du régime narratif actualisé. À l'inverse des novellisations typiques qui sont toutes des écritures « sans auteur $»^{9}$, censées restituer avec des mots l'effet d'illusion diégétique créé par les images, Cinéma adopte la narration homodiégétique. C'est un spectateur qui raconte ici son film préféré, en vue non pas d'en fournir seulement un résumé détaillé, mais pour rendre compte avec des mots de «l'art de Sleuth» $(C$, p. 122) : dire la complexité de l'œuvre qui l'a émerveillé plus que toute autre. Même s'il le fait avec le plus d'exactitude possible, sa présence dans le texte fait infraction aux principes de la novellisation littéraire. D'ailleurs, l'objectif même du récit entraîne des dérogations par rapport à la structure du film. Puisque le langage s'avère impuissant à dire les images et que le résumé seul ne suffit pas à refléter la perfection de Sleuth, il est impossible au narrateur d'éviter des achronologies et des métacommentaires qui, tout en éclaircissant le sens de l'œuvre, défigurent sa structure originelle. En apparence une novellisation littéraire, le roman de Viel est plutôt le récit d'une expérience cinématographique que celui du film même qui illustre les relations affectives entre l'homme et l'art.

Que l'expérience artistique est une des formes très singulières de l'amitié, les philosophes l'ont montré plus d'une fois. Selon John Dewey, seule une relation intime avec une œuvre d'art, pareille à celle que l'on établit avec un ami, permet d'en saisir le sens ainsi que le sens du monde qu' elle renferme. Pour Marcel Proust, l'art est un intermédiaire incontournable dans les relations humaines et constitue

9 J. Baetens, « La novellisation contemporaine en langue française », <http://www.fabula.org/ lht/2/Baetens.html> [consulté le 31.03.2016]. 
la seule façon de tisser des liens entre les hommes, essentiellement séparés ${ }^{10}$. C'est justement cet attachement à l'art que conte Cinéma, tout en démontrant sa puissance irrésistible et des incidences ambiguës. Sleuth est bien une source d'émotions, si intenses que le narrateur s'efforce de les capter une par une dans un carnet réservé à cet effet, afin de pouvoir les revivre ensuite. Car rien d'autre ne l'émerveille à tel point que ce film auquel il voue une admiration exceptionnelle, le glorifiant dans des commentaires enthousiastes, le valorisant à l'extrême par des adjectifs mélioratifs : grand, formidable, épuré. Or, la personnification du film en dit long sur le caractère des émotions éprouvées par le narrateur, qui relèvent plus d'une fascination pathologique que d'un ravissement pour une œuvre d'art parfaite :

Je n'ai pas de vie à côté du film, je suis un homme mort sans Sleuth, oui, Sleuth, le titre original du film en anglais, pour moi ce n'est pas un nom de film, c'est le nom d'un ami, je dis Sleuth, comme je dirais Andrew. Quelquefois je sors de chez moi et je m'excuse auprès de Sleuth parce que je le laisse seul, et je fais très attention où je l'entrepose, loin du froid, loin de la chaleur, et je le salue quand je rentre. $(C$, p. 96$)$

Si l'expérience artistique consiste en « une identification de nous-mêmes avec la beauté qui se trouve dans une pensée, une action ou une personne qui n'est pas la nôtre » ${ }^{11}$, cette identification se fait dans le cas de Cinéma totale et absolue. « Tout ce que vous faites à Sleuth, c'est à moi que vous le faites » $(C, \mathrm{p} .117)$, voilà le témoignage personnel d'une fusion abusive qui se produit dans le psychisme du spectateur traqué par l'image. Subjugué par le thriller de Mankiewicz, le narrateur ne cesse de le visionner « dans tous les sens, en toutes situations, et le confronter avec mon monde à moi, mon réel à moi qui change tout le temps, pour tester la résistance du film à mon mental » $(C, \mathrm{p} .37)$. Et si le film résiste avec obstination aux éclaircissements rationnels, le psychisme du narrateur se laisse piéger par la force de l'illusion. Tantôt, il se reconnaît dans les protagonistes fictifs et s'investit dans leurs jeux pervers, tantôt eux-mêmes prennent du relief et transparaissent dans les gens qu'il croise au quotidien. L'univers filmique s'infiltre dans sa vie jusqu'à se confondre avec le réel, et c'est la réalité de Sleuth qui devient la sienne propre.

Basé sur le transfert intersémiotique d'une œuvre écranisée en un livre, le roman de Viel est, certes, une expression de l'hommage de l'écrivain envers le cinéma en général, qui a toujours été sa passion et sa source d'inspirations littéraires, et envers la réalisation de Mankiewicz en particulier, envers un film d'une complexité virtuose. Or, si l'amitié est le mobile de l'écriture, elle est aussi son enjeu thématique majeur, car l'écrivain s'attache surtout à illustrer dans la fiction cette relation émotionnelle entre l'homme et l'art que Dewey et Proust ont théorisée dans leurs écrits. À cette différence près qu'il fait ressortir plutôt son côté

${ }^{10} C f$. M. Proust, «Sur la lecture », [dans :] J. Ruskin, Sésame et les lys, Éditions Complexe, Bruxelles 1987, pp. 37-97.

11 J. Dewey, Art as Experience. Euvres philosophiques III, Publications de l'Université de Pau / Éditions Farrago, Tours 2005 [1934], p. 399. 
sombre, en démontrant comment, d'une manière particulièrement sournoise, l'art effectue une transformation négative du moi et sépare l'individu de la vie réelle au lieu de lui permettre d'en faire une expérience épanouissante. Une amitié trop fusionnelle avec l'art se termine par une aliénation.

Version littéraire des films de gangsters, L'Absolue perfection du crime pousse à l'extrême le travail de recyclage des poncifs génériques. Fondé sur un motif surexploité par les metteurs en scène, celui du braquage raté d'un casino, le roman reprend fidèlement le scénario classique du coup monté et il rejoue, à tous les détails près, les scènes cinématographiques stéréotypées telles que les retrouvailles de vieux amis sortis de prison, l'arnaque aux jeux de hasard ou la folle course-poursuite du traître par tous les moyens de transport possibles et imaginables. L'on voit bien que pour Viel «écrire ce ne serait jamais alors tout à fait écrire mais recueillir $»^{12}$, à savoir reprendre dans le texte littéraire des scénarios préexistants et des scripts tout faits pour en tisser une intrigue romanesque. L'Absolue perfection du crime se présente comme une condensation littéraire des films de gangsters, une sorte d'encyclopédie livresque du genre cinématographique qui renferme dans ses pages un ensemble d'épisodes caractéristiques des fictions mafieuses. Récupérant l'imaginaire du genre, le roman contrevient toutefois à ses conventions tant au niveau diégétique qu'au niveau formel. Lassés de leur vie criminelle avec laquelle ils n'ont pourtant pas la force de rompre à cause de leur passivité existentielle — « si soi-même on veut seulement survivre, on continue » $(A P C, \mathrm{p} .24)$ - les protagonistes du roman ne sont que des caricatures minables des vrais gangsters, et le rythme de l'action, retardé par les pressentiments d'un échec, n'a rien de séduisant. Les subversions formelles s'effectuent par des jeux de niveaux ontologiques : centrale dans le texte, la scène du casse au casino s'avère n'être qu'une reconstruction théâtrale des événements à l'usage du procès au tribunal qui les suit. Le dénouement est révélé bien avant la fin et l'effet d'illusion romanesque - crucial dans le cas des fictions fondées sur le suspense - dissipé par ce glissement du spectacle à la place du réel.

À l'instar des fictions basées sur le motif de la trahison, L'Absolue perfection du crime pose avec insistance le problème des relations humaines, de leur complexité et leurs motivations. Dans le monde mafieux, l'amitié joue un rôle fondamental, celui d'un instrument de protection et d'alliance censé fortifier la position sociale. Le texte laisse bien voir la force de la collectivité ne serait-ce que dans la scène de beuveries nocturnes dans le bar où, du fait de « se montrer en ville le soir, vestes noires, cheveux peignés comme il faut, parfums, [...] on se sentait observés, c'est-à-dire respectés » $(A P C$, p. 59). Or, les relations calculées n'aboutissent pas à des attachements réels et profonds. Émergeant dans la conscience des membres de la bande sous l'effet des paroles de son chef et soigneusement entretenue au cours des rencontres habituelles à sa maison, censées former l'esprit de clan, la

12 J. Faerber, «Le livre aveugle ou la passion anthologique dans l'œuvre de Tanguy Viel », Relief 6 (2), 2012, p. 80. 
conviction qu' ' on était plus liés qu'à s'imaginer du même sang » $(A P C$, p. 18) se révèle totalement fausse. La famille des gangsters n'est qu'une pure illusion, un lieu d'isolement plutôt qu'un terrain de rapprochement, " une communauté sans communauté $»^{13}$, pour citer Jacques Derrida, qui laisse chacun dans sa propre solitude. Car dans l'univers mafieux, où la valeur de l'individu est strictement corrélée à son utilité dans les combines illicites, il est risqué d'être soi-même, d'avouer ses opinions ou émotions. L'épisode du retour d'une visite chez l'oncle est particulièrement révélateur de l'ambiance feutrée de sujets tabous et de nondits qui règne dans ce monde impitoyable :

Les relations qu'on entretenait ensemble, ou séparément, chacun, l'oncle, la tante, Marin, Andrei, moi, gardant pour soi le nœud qui nous plongeait dans cet état de fausse méditation, où les paroles dites avec précaution, avec parcimonie, avec ce ton aussi qui voulait qu'on attende toujours un éclairage des mots, où ces paroles, donc, laissaient seulement supposer la partie immergée de l'iceberg. (APC, p. 21)

L'absence du dialogue, qui est pourtant une condition indispensable de l'amitié, empêche la proximité réelle. La gêne et la crainte paralysent les relations interpersonnelles, et les seuls moments de complicité totale ne se vivent qu'un verre à la main au zinc du Lord Jim : « il n'y a jamais que là-bas qu'on a pu se sentir si proches, si amis, si fraternels » $(A P C$, p. 60). « L'utile, l'intérêt n'a rien de fixe ; [...] l'amitié disparaît aussi rapidement, avec la seule cause qui l'avait formée ${ }^{14}$ - c'est justement selon ce scénario décrit autrefois par Aristote qu'évolue la relation calculée de L'Absolue perfection du crime. La trahison a lieu, d'autant plus vilaine que préparée avec préméditation par le futur chef de la bande et l'auteur même de l'idée du dernier casse. La poursuite du Judas par le narrateur, objet de la dernière partie du roman, ne vise pourtant pas au seul règlement de comptes et son motif est plus émotionnel que matériel. Il le rend responsable d'avoir détruit cette famille qu'ils ont formée ensemble, famille qui - si imparfaite et assujettissante soit-elle - investissait de sens la vie de tous ses membres, incapables de mener une existence autonome, et était « le sésame de [leur] identité » ${ }^{15}$.

Outre la forme romanesque travaillée par des variations ludiques sur les sousgenres littéraires, la trilogie de Viel s'adapte aux canons de la littérature contemporaine par l'emploi de la narration non-fiable, catégorie plutôt rare dans le roman traditionnel qui privilégie surtout la figure du narrateur autoritaire, aux compétences cognitives et discursives idéales ${ }^{16}$. Comme le constatent Frances Fortier

13 J. Derrida, Politiques de l'amitié, Éditions Galilée, Paris 1994, p. 62.

14 Aristote, Éthique à Nicomaque, Le Livre de poche, Paris 1992, p. 331.

${ }^{15}$ S. Chaudier, J. Négrel, « Tanguy Viel : l'empire des passions tristes », La revue internationale des livres et des idées 12, juillet-août 2009, p. 54.

16 Très fréquente dans la littérature contemporaine, la narration non-fiable n'est pas, certes, son privilège ni invention. On peut en déceler des occurences dans le roman picaresque et la littérature fantastique où le récit est à la charge de narrateurs faillibles, perdus dans la réalité qu'ils ne comprennent pas ou n'arrivent pas à saisir. Elle est particulièrement répandue dans la littérature de l'époque de modernité, aussi bien dans celle de l'avant-garde qui multiplie les narrateurs 
et Andrée Mercier, " plusieurs romans contemporains [...] problématisent leur situation de transmission narrative, mais sans sacrifier pour autant leur dimension romanesque $»^{17}$. Chez Viel, c'est même une étroite corrélation de ces deux éléments que l'on peut observer, la narration non seulement configurant l'intrigue de façon à capter l'attention du lecteur mais constituant une sorte d'appendice à l'histoire racontée. Car la manière de raconter les événements développe et approfondit selon ses propres moyens le motif de l'amitié, central dans les trois romans. Qui plus est, la non-fiabilité du narrateur ne découle pas tant d'un défaut de compétences ou de manipulations stratégiques, même si ces cas classiques sont actualisés surtout dans Le Black Note, que des troubles affectifs touchant la sphère relationnelle. Les romans analysés reposent tous les trois sur un schéma identique : le récit est pris en charge par un narrateur homodiégétique qui, a posteriori, raconte l'histoire d'une amitié intense et complexe qu'il a vécue. Assez classique, cette situation énonciative met à jour un dispositif narratif tout à fait ingénieux et profondément associé à l'intrigue romanesque : assuré par le narrateur unique, le récit évolue vers une plurivocalité ${ }^{18}$ très particulière, et la motivation narrative qui est de relater les faits se double d'une visée dialogique.

Tout en instaurant ce dispositif qui forme une sorte de « clef de voûte ${ }^{19}$ narrative de la trilogie analysée, Le Black Note en présente également la forme la plus sophistiquée. Le récit prend la forme d'un long monologue mené obsessivement par le narrateur pour occuper ses longues journées à la clinique psychiatrique. Monologue singulier, pas tout à fait le sien propre, car c'est avec les mots des autres qu'il est tissé plutôt que par ses propos. Dans sa parole cahotante retentissent les paroles de ses amis et surtout ceux du leader du groupe, Paul, mort tragiquement dans l'incendie. Il ne s'agit pas là seulement d'une réaction discursive au traumatisme vécu, piste suggérée à plusieurs reprises dans le texte par les images de visions cauchemardesques rongeant tant le cerveau que le langage du narrateur : "C'est Paul qui parle en moi, le même Paul qui est mort, c'est à cause du poids qu'il occupe dans mon crâne, alors il remonte à la surface et je dis

retors et manipulateurs que dans celle focalisée sur la problématique de divers traumas psychiques où l'on a affaire aux narrateurs en déficit de savoir ou incapables de raconter, parfois aussi à une pluralité de narrateurs et les narrations démultipliées, incohérentes et contradictoires (cf. M.-P. Huglo, S. Rocheville (dir.), Raconter? Les enjeux de la voix narrative dans le récit contemporain, L'Harmattan, Paris 2004).

${ }^{17}$ F. Fortier, A. Mercier, « La narration impossible. Conventions réalistes, catégories narratologiques et enjeux esthétiques », [dans :] F. Fortier, A. Mercier (dir.), La transmission narrative. Modalités du pacte romanesque contemporain, Éditions Nota bene, Québec 2011, p. 334.

${ }^{18}$ La notion de plurivocalité est avancée par Mikhaïl Bakhtine dans Problèmes de l'ceuvre de Dostoïevski [1929] pour désigner un entrelacement de diverses voix dans le roman, celles de l'auteur, du narrateur et des personnages, qui se joignent de sorte à engendrer une structure polyphonique complexe. Le roman plurivocal n'interpose pas la domination d'une seule conception du monde mais laisse voir une pluralité d'univers idéologiques à statut égal dans le texte.

19 J. Faerber, op. cit., p. 84. 
n'importe quoi » (BN, p. 101). Composé de phrases prononcées autrefois par le prétendu jazzman, le monologue du narrateur traduit sa profonde dépendance psychologique de son ami et dévoile le mécanisme par lequel elle s'est produite. C'est grâce à d'étonnantes capacités rhétoriques que Paul a réussi à l'enjôler, ainsi que ses compagnons, en peignant avec des mots éloquents une vision séduisante d'un succès musical et d'une communauté parfaite qu'ils formeraient tous ensemble :

[...] il parlait si bien, et la tonalité de sa voix toujours, le tremblement qu'il effectuait pour pondérer si bien le discours, la prophétie du langage qu'il dégageait, avec ce droit qu'on a, quand on est l'aîné, de poser les lèvres avec intention d'émouvoir, alors on y croyait [...]. ( $B N, \mathrm{p} .41)$

Ces mots pleins de force et de vitalité ont soumis les autres à sa volonté et les ont fait vivre selon ses fantasmes illusoires. Ils résonnent désormais dans la tête du narrateur, incapable de se libérer de l'emprise de son ami toxique même après sa mort et de s'exprimer autrement qu'en empruntant ses « grandes phrases » $(B N$, p. 27). Si, comme le constate Faerber, « la puissance d'un personnage, d'un frère sombre, est avant tout discursive $»^{20}$, c'est au gré d'un discours infirme du narrateur qu'elle se voit finalement dénoncée et révélée comme principal motif du crime advenu.

S'il traduit, par son caractère plurivocal, la face sombre de la relation amicale expérimentée par le narrateur, le monologue reflète également la valeur exceptionnelle qu' elle a eue pour lui. Dès l'incipit, on remarque une dimension nettement dialogique du discours narratorial qui se laisse voir dans « l'orientation de la parole du 'discoureur' anonyme vers le destinataire ${ }^{21}$ voire vers plusieurs destinataires qui sont, à tour de rôle, interpellés par le narrateur. Ce n'est pas la forme d'un soliloque que prend le premier roman de Viel mais celle d'une suite de conversations avec divers interlocuteurs dont les compagnons du Black Note, un nouveau collègue, comme lui patient de la clinique psychiatrique, et le directeur de l'établissement. Ces entretiens sont imprégnés d'une profonde ambiguïté et visent autant à dire qu'à taire ce qui s'est passé : ils présentent, chacun, une version divergente des faits de manière à permettre de reconstituer l'histoire tragique de l'amitié inspirée par la passion musicale tout en masquant l'identité du criminel. Cherchant à tout prix à dissimuler la vérité par ses propos contradictoires qui font obscurité, c'est son ami qu'il tient à protéger, le meurtrier réel qui reste en liberté. Ce désir de protection alimente également la pulsion du dire, le récit se posant pour objectif de restaurer avec des mots cette vie communautaire qui a définitivement pris fin et dont le souvenir s'estompe doucement avec le temps qui passe ainsi que sous l'effet des médicaments. Car pour le narrateur " oublier c'est pire, et tu n'as rien pour t'accrocher » $(B N$, p. 101), comme il le dit à un camarade. La vie vécue ensemble à Black Note,

${ }^{20}$ Ibidem, p. 83 .

${ }^{21}$ F. Wagner, "C'est à moi que tu parles ? (Allocutaires et auditeur dans Le Black Note de Tanguy Viel) », [dans :] S. Houppermans, Ch. Bosman Delzons, D. de Runter-Tognotti (dir.), Territoires et terres d'histoires. Perspectives, horizons, jardins secrets dans la littérature française d'aujourd'hui, Rodopi, Amsterdam-New York 2005, p. 227. 
avec ses hauts et ses bas, constitue un épisode particulièrement important pour lui, une expérience de rapprochement des autres qui confère un sens à l'existence. Au moyen des conversations avec ses compagnons d'autrefois, dont on ne sait pas si elles se produisent réellement ou ne se déroulent que dans l'imagination du narrateur, ce sont tous ces moments de joie et de tristesse qu'il veut revivre encore une fois pour ressentir les émotions d'une amitié même si elle était imparfaite.

La même double narration, à la fois plurivocale et dialogique, est actualisée dans Cinéma de manière plus discrète. Le texte ne contient pas d'indications typiques d'emprunts discursifs telles que les guillemets ou les italiques, mais fait se relayer deux régimes narratifs - la narration neutre de type hétérodiégétique, qui retrace la réalité filmique, et la narration subjective homodiégétique, où la figure du narrateur transparaît - de manière à produire l'effet de deux voix qui parlent à tour de rôle. Cette plurivocalité est, d'ailleurs, thématisée dans le texte même dans l'un des multiples passages où le narrateur se sent obligé de justifier sa démarche par la complexité de son projet :

[...] toutes ces choses si visibles, je dois les laisser parler à ma place, non pas à ma place, je suis là aussi, mais ensemble, que les images et moi on parle ensemble, voilà ce que je dois faire [...]. Moimême, si j'étais raisonnable, je ferais une version épurée, je ferais les coupes dans le discours si j'étais raisonnable, j'arrêterais de gaspiller, comment dire, de la pellicule vocale. ( $C$, p. 43)

Son intention étant de raconter le film qui l'a fasciné, c'est au résumé le plus fidèle possible de l'intrigue qu'il se livre, s'effaçant de son récit pour donner la priorité à la parole de l'œuvre d'art préférée et laisser parler Sleuth seul à travers ses épisodes successifs. Ce renoncement intentionnel à son propre langage est tout à fait significatif dans la mesure où il traduit « la perte de soi dans la domination de l'autre $»^{22}$. Convaincu de la perfection absolue de l'œuvre culte, le narrateur manifeste un évident manque de confiance en ses propres capacités discursives et n'ose pas parler autrement qu'au moyen de citations empruntées au film. Or, étant donné l'inefficacité du langage par rapport aux images filmiques, il n'a d'autre choix que d'intervenir pour dire ce que le résumé seul est incapable de révéler. Sa voix se joint désormais à celle de Sleuth, s'empêtre entre les scènes et les dialogues avec des commentaires, des explications et des analyses censées éclaircir les ambiguïtés de l'intrigue ou faire ressortir les détails significatifs. Jamais autonome, elle est toute entière subordonnée à la voix du film, cherche à la seconder pour énoncer cette réalité fascinante composée avec des images. Il est impossible, également, de ne pas remarquer sa profonde impuissance face au langage visuel du film qu'elle n'arrive pas à traduire avec des mots et des phrases. "C'est vanité de faire parler les images » $(C$, p. 43$)$, constate avec désespoir le narrateur dont les efforts discursifs n'atteignent pas l'objectif posé.

${ }^{22}$ S. Rongier, « Tanguy Viel : une stratégie de l'effrangemement [sic!] », <http://remue.net/ cont/viel_cinema.html> [consulté le 31.05.2016]. 
La dimension dialogique de Cinéma ne se laisse pas facilement remarquer du fait que le texte n'investit pas la forme conversationnelle, le narrateur ne s'adressant pas ici directement à quelque interlocuteur concret. Le but de son long monologue dépasse pourtant largement la seule fonction représentative. Les questions qui parsèment régulièrement le texte, les doutes exprimés çà et là sur la clarté de ses propos ainsi qu'un acharnement explicatif visible dans le souci du détail, les épanorthoses constantes et les ajouts interminables traduisent la réelle intention du narrateur qui est de révéler la grandeur de Sleuth au monde entier. À ceux qui ne le connaissent pas encore et qu'il invite aux projections doublées d'exégèse, ainsi qu'aux mauvais spectateurs dont l'indifférence face à l'œuvre qu'il juge grandiose l'indigne par-dessus tout. Ces derniers surtout se voient interpellés de biais dans le texte, dans les passages qui décrivent les réactions du public manifestement incapable d'apprécier ce qui se passe sur l'écran. Laborieusement effectué tout le long du texte, le travail d'herméneutique est dicté par les émotions qu'il ressent et qu'il veut absolument partager avec les autres. Selon lui, il faut « comprendre pour aimer » $(C$, p. 75$)$ et c'est justement ainsi qu'il procède, avec l'espoir de susciter chez ceux à qui il adresse sa parole une admiration envers Sleuth analogue à la sienne. Il est très intéressant de remarquer que ses efforts pour partager avec l'entourage une fascination artistique ne relèvent pas d'une recherche d'amitié avec des gens, mais sont entièrement subordonnés à son amour filmique : le narrateur n'éprouve aucun besoin de rencontrer des âmes fraternelles pour briser sa solitude de spectateur invétéré, qui ne le gêne pas du tout, mais ce sont des amis à Sleuth qu'il veut trouver, pour qu'ils applaudissent toutes ses qualités. Plus le film échappe à la glose langagière, plus le narrateur se fait autoritaire et sa fascination tourne au fanatisme : « Moi je trouve ça extraordinaire, tout le monde doit trouver ça extraordinaire, et beau, et grand, tout le monde, n'importe où dans l'univers, c'est irréfutable » ( $C$, p. 119). Incapable d'inspirer chez les autres cette affection esthétique qu'il porte au film, il tente de l'imposer par des consignes prescriptives en dépit de ce que, selon les philosophes, l'« amitié n'est jamais que le résultat d'une décision réfléchie $»^{23}$ et surtout personnelle.

Le dispositif narratif de L'Absolue perfection du crime fait écho à celui du Black Note sans pourtant le reproduire complètement. Tout en racontant l'histoire de sa vie mafieuse, le narrateur ne cesse de rapporter les propos de Marin, initiateur du casse au casino. Propos fallacieux et sournois censés séduire pour détruire, car s'ils ont déployé la vision excitante du " coup de leur vie », ce n'est rien que pour induire tout le monde en erreur. Leur puissance est d'autant plus irrésistible qu'elle est redoublée : prononcés par l'homme le plus fort du groupe, « rayonnant» d'énergie $(A P C$, p. 34) de manière à dominer les autres, ils bénéficient d'une approbation inconditionnelle du vieux chef qui les sanctionne de son autorité, ou ne font que rejoindre son éternel discours en reproduisant adroitement les sentences du décalogue mafieux imposé par le parrain :

${ }^{23}$ Aristote, Éthique..., p. 338. 
[...] il faut durer encore un peu, ajoutait l'oncle, rester loyal au milieu des traîtres. Des traîtres, oui, insistait Marin, marchant sans cesse sur le parquet grinçant, râpé, et se faisant l'écho des paroles de plus en plus ternes, de plus en plus maigres de l'oncle vieillissant. (APC, p. 22)

Dans L'Absolue perfection du crime, la narration est moins « phagocytée ${ }^{24}$ par les mots de l'autre que composée intentionnellement avec leur concours. Si le récit se fait majoritairement au discours rapporté, c'est que le narrateur manifeste une sorte d'impuissance discursive causée par ses relations sociales : voué au silence qui est une règle d'or dans l'univers mafieux, il n'a jamais osé ni contredire son puissant ami ni même exprimer ses propres pensées. Désarmé par ce mutisme continu en dépit de sa faculté de parler, il n'a d'autre choix que d'évoquer toute cette liste « de saintes maximes et d'adages $»^{25}$ que Marin a proférés avec un « air de prêtre » $(A P C$, p. 28) pour dire la manière dont ils ont façonné son propre personnage et sa vie.

C'est à ce Marin qu'il ne cesse de citer que le narrateur adresse son monologue, en l'interpellant régulièrement par son nom. Autorisé à parler au cours du procès où il est principal témoin, il tente d'établir ce dialogue qu'il n'a jamais pu ouvrir auparavant de crainte d'enfreindre les lois du milieu. Son intention est autant de dire enfin ce qu'il n'a jamais osé avouer que de s'enquérir des motivations qui ont guidé son ami. En lui expliquant ses doutes sur la réussite du projet et en les justifiant par son pressentiment jamais révélé qu'il était impossible de faire perdurer l'empire déclinant de l'oncle qui, « au fond, mon vieux Marin, [...] s'en foutait de tes projets de dingue » $(A P C$, p. 62), il parvient à comprendre son hypocrisie et le sens réel de ces mots si trompeurs : «L'absolue perfection du crime». Établi à titre posthume, rien que dans l'imagination du narrateur après qu'il a tué Marin, ce dialogue où se voient élucidés les non-dits entre les protagonistes est en même temps un discours d'adieu adressé à l'ami d'autrefois. Non satisfait de se venger, le narrateur éprouve un irrésistible besoin d'exprimer avec ces mots qu'il a dû si longtemps retenir sa profonde déception face à une amitié qui n'était qu'une illusion. C'est pour se libérer enfin de son emprise corrosive qu'il dénonce, avec des paroles encore hésitantes, celui qui a joué avec les idéaux pathétiques de la famille et de la fraternité pour abuser de la confiance de ses proches. Sa première et à la fois dernière conversation avec Marin scelle ainsi l'acte du meurtre, marque le détachement définitif du narrateur par rapport au passé et ouvre une nouvelle étape dans sa vie.

Plus qu'une seule fiction organisée autour du motif de l'amitié, la trilogie de Viel se présente comme une réflexion sur les relations affectives que l'homme développe durant sa vie. L'emploi des modalités de narration contemporaines telles que la réécriture subversive des genres et la narration non-fiable sert, certes, à adapter le récit aux canons romanesques de l'époque dite de la désillusion époque de crises multiples qui ont ébranlé la conviction du « sens collectif de

\footnotetext{
${ }^{24}$ F. Wagner, op. cit., p. 230.

25 J. Faerber, op. cit., p. 84.
} 
l'avenir qui vaut pour l'humanité entière $»^{26}$ — mais elle vise surtout à restructurer le motif de la désillusion personnelle, si présent dans la littérature, et à éviter un pathos mélodramatique. Inscrits dans les formes génériques propres à la culture populaire dont ils détournent les motifs typiques et les intrigues conventionnelles, les trois romans récusent l'idée de la captatio illusionis, fondement du roman classique, non pourtant pour sombrer dans la parodie mais pour toucher à l'universel. La trilogie de Viel est à lire comme une « fable du sens $»^{27}$ sur la vie émotionnelle de l'homme, son insoutenable besoin de l'autre et sa peur panique de la solitude. C'est un panorama relationnel que l'auteur établit, chaque texte illustrant un autre type d'attachement parmi ceux énumérés déjà par Aristote dans sa célèbre Éthique à Nicomaque, dont on voit qu'elle n'a rien perdu de son actualité et se prête toujours bien à la réflexion sur l'amitié. Histoire d'une communauté musicale formée par des passionnés de jazz, Le Black Note fait voir la forme la plus idéale de l'amitié, celle qui unit les gens « qui se ressemblent par leur vertu $»^{28}$; Cinéma - qui raconte une fascination artistique - représente la relation née d'un plaisir esthétique ; L'Absolue perfection du crime - récit d'intrigues mafieuses - décrit une liaison calculée qui « se forme par une vue d'intérêt et d'utilité ${ }^{29}$. La narration, originale, tissée de mots des amis et organisée comme une conversation mentale, fait ressortir d'autant plus la force à la fois bénéfique et toxique de cette amitié dont le récit conte les aléas.

\section{NARRATING PERSONAL DISILLUSIONMENTS IN THE ERA OF DISILLUSIONMENT: TANGUY VIEL AND CONTEMPORARY FORMS OF FRIENDSHIP STORY}

Summary

Friendship is one of the key motifs in Viel's work. Its importance is highlighted especially in his early novels. In his exploration of this eternal problem of a relationship with another person, Viel departs from typically realistic novelistic conventions and combines subversive rewriting of genres, typical of contemporary fiction, with unreliable narration, manifested in a peculiar multivocality. The aim of this article is to analyse this textual duality and the manner in which it contributes to the creation of a touching story about human friendships.

Key words: Tanguy Viel, friendship, genre, generic variations, unreliable narration.

${ }^{26}$ A. Cousseau, « Postmodernité : du retour au récit à la tentation romanesque », [dans :] B. Blanckeman, A. Mura-Brunel, M. Dambre (dir.), Le roman français au tournant du XXI siècle, Presses Sorbonne Nouvelle, Paris 2005, p. 360.

27 T. Viel, « Seconde main », <http://recitsentreamis.over-blog.com/article-seconde-main-entretien-avec-thomas-clerc-et-tanguy-viel-73672781.html> [consulté le 31.05.2016].

28 Aristote, Éthique..., p. 331.

${ }^{29}$ Ibidem, p. 335. 